OPEN ACCESS

Edited by:

Selin Kara,

Technical University of Hamburg,

Germany

Reviewed by:

Kathrin Castiglione,

Technische Universität München,

Germany

Thierry Michon,

Institut National de la Recherche

Agronomique, France

*Correspondence:

Jan von Langermann

jan.langermann@uni-rostock.de

Specialty section:

This article was submitted to Microbiotechnology, Ecotoxicology

and Bioremediation,

a section of the journa

Frontiers in Microbiology

Received: 12 September 2017

Accepted: 16 October 2017

Published: 09 November 2017

Citation:

Uhrich D and von Langermann J (2017) Preparation

and Characterization of Enzyme

Compartments in UV-Cured

Polyurethane-Based Materials

and Their Application in Enzymatic

Reactions. Front. Microbiol. 8:2111.

doi: 10.3389/fmicb.2017.02111

\section{Preparation and Characterization of Enzyme Compartments in UV-Cured Polyurethane-Based Materials and Their Application in Enzymatic Reactions}

\author{
Diana Uhrich and Jan von Langermann* \\ Biocatalysis Group, Institute of Chemistry, University of Rostock, Rostock, Germany
}

The preparation and characterization of UV-cured polyurethane-based materials for the mild inclusion immobilization of enzymes was investigated. Full curing of the polymer precursor/enzyme solution mixture was realized by a short irradiation with UV-light at ambient temperatures. The included aqueous enzyme solution remains highly dispersed in the polymer material with an even size distribution throughout the polymer material. The presented concept provides stable enzyme compartments which were applied for an alcohol dehydrogenase-catalyzed reduction reaction in organic solvents. Cofactor regeneration was achieved by a substrate-coupled approach via 2-propanol or an enzyme-coupled approach by a glucose dehydrogenase. This reaction concept can also be used for a simultaneous application of contrary biocatalytic reaction conditions within an enzymatic cascade reaction. Independent polymer-based reaction compartments were provided for two incompatible enzymatic reaction systems (alcohol dehydrogenase and hydroxynitrile lyase), while the relevant reactants diffuse between the applied compartments.

Keywords: biocatalysis, immobilization, alcohol dehydrogenase, inclusion, organic solvent

\section{INTRODUCTION}

Enzymes are highly versatile biocatalysts that catalyze a vast variety of reactions under typically mild reaction conditions (Faber, 2004; Tao and Kazlauskas, 2011). In their natural environment these enzymes are also often localized within cellular or subcellular domains (microenvironment compartmentalization), which provides optimal conditions for the respective reaction, e.g., $\mathrm{pH}$ and availability of (co-)substrates, cofactors, etc. (Ovádi and Saks, 2004; Zecchin et al., 2015). This spatial organization facilitates also an easy transfer of metabolites from one catalytic domain to the next, yielding an effective assembly line with increased overall output of the desired product. For example, eukaryotic cells are based on a complex of membranes and other compartments, which are specialized for the required biological functions (Huh et al., 2003; Barabási and Oltvai, 2004). These compartments are not necessarily permanent, as found in the metabolic cycle of yeast, which features temporal compartments (Tu et al., 2005). In general, such permanent and temporal compartments allow within a complex orchestra of reaction pathways biological systems to perform anabolic and catabolic processes in a coordinated approach 
with an effective usage of resources. In contrast, the use of enzymes for synthetic purposes frequently requires nonphysiological reaction conditions, e.g., use of an organic solvent or extreme $\mathrm{pH}$, which are not compatible with classical natural compartments. For this purpose tailor-made artificial compartments are required that provide sufficient applicability under non-conventional conditions. The enzyme remains similarly to their natural analogs segregated from the external solution within the compartment. However, such artificial concepts require (a) a careful control of the preparation process to facilitate catalytically active enzymes in the nonnatural compartments, (b) sufficient chemical and mechanical stability at the chosen non-conventional reaction conditions, and (c) a relatively low diffusion limitation of the reactants through the respective support material to avoid bottlenecks in the synthesis process. Currently a rather limited selection of synthetic compartmentalization options is available for such purposes, which include predominantly the application of solgels, silicone-based "static emulsions" and less frequently the use of full solution inclusions/cell mimicry, e.g., in polysiloxane and polymersomes (Pierre, 2004; Buthe et al., 2005; AnsorgeSchumacher, 2007; Wu et al., 2011; Zhang et al., 2011; Patterson et al., 2014; Klermund et al., 2017). Unfortunately sol gelconcepts include relatively harsh conditions, e.g., the use of alkylsilanes and at least one drying processes, which alters the apparent matrix and thus often affects the compartmented enzyme negatively (Cabirol et al., 2006). An inclusion of an entire aqueous phase in a compartment is in theory preferred since the enzyme remains in the aqueous solution, but requires often complex and time-consuming preparation procedures.

In this study, we present a simplified and very fast approach to enzyme compartments via UV-curable polymer preparations and their use for synthetic purposes (Norland_Products, 2017). Herein a hydrophobic polyurethane precursor is mixed with an aqueous enzyme solution forming a viscous emulsion, which is then solidified by UV-irradiation within ca. $5 \mathrm{~min}$ at ambient temperature (Carlborg et al., 2011). In the resulting solid material the original aqueous enzyme solution remains as small droplets dispersed in the polymer matrix (Figure 1). The obtained solid material is eventually cut or grinded into the desired size to minimize potential reactant diffusion limitations through the polymer network. The alcohol dehydrogenase-catalyzed reduction of prochiral ketones to the respective chiral alcohols was chosen as a model reaction for applicability.

Moreover, enzymes usually operate at relatively similar reaction conditions and a concurrent application of more than one isolated enzyme in the same reaction vessel is obvious. Consequently numerous process concepts were reported for synthetic purposes (Schrittwieser et al., 2011; Sánchez-Moreno et al., 2012; Guterl and Sieber, 2013; Oberleitner et al., 2013; Oroz-Guinea and García-Junceda, 2013; Riva and Fessner, 2014; Muschiol et al., 2015). In general, four different designs of enzymatic cascade (tandem) reactions are possible: linear, parallel, orthogonal, and cyclic cascade (Ricca et al., 2011; Simon et al., 2014). Examples include, aside the above mentioned multi-enzymatic approaches, numerous variations of organoor (transition) metal catalysts in combination with biocatalysts
(Gelman et al., 2002; Köhler and Turner, 2014). The use of such reaction cascades typically facilitates three main advantages: (a) no need to isolate intermediates, which significantly reduces efforts in downstream processing and improves overall yield, (b) avoiding inhibiting or toxic concentrations of intermediates, and (c) overcoming thermodynamically unfavored reaction equilibria by pushing the reaction to the product side, which again improves overall yield of the process (Ricca et al., 2011; Abu and Woodley, 2015). On the other hand, the presence of multiple reagents in one solution may cause unwanted secondary (catalytic) side reactions and thus a careful selection and control of the reaction process is still required. Fortunately, compartmentation of reaction zones can be used to (partly) overcome certain limitations of such (bio)catalytic cascade reactions since optimal reaction conditions for each (bio)catalytic reaction system can be selected. Thus, the presented polyurethane-based material was used for the preparation of two different, non-compatible aqueous conditions and subsequently used in a biocatalytic cascade reaction. As a model reaction a combination of two very contrary reaction systems was chosen, the alcohol dehydrogenase from Lactobacillus kefir, LkADH and hydroxynitrile lyase from Manihot esculenta, MeHNL.

\section{MATERIALS AND METHODS}

\section{General}

Acetophenone, aliphatic ketones, 2-propanol, triethanolamine (TEA), citric acid, dipotassium phosphate, and methyl tertbutyl ether (MTBE) were obtained from Sigma-Aldrich, Seelze, Germany. Magnesium chloride, potassium dihydrogen phosphate, mandelonitrile, glucose, and concentrated hydrochloric acid were purchased from Merck, Darmstadt, Germany. Sodium cyanide was received from Fluka Chemika AG, Buchs, Switzerland. In addition, mandelonitrile was stored at $-18^{\circ} \mathrm{C}$ to minimize decomposition to benzaldehyde and hydrogen cyanide. Sodium hydroxide was purchased from VWR International, Darmstadt, Germany and calcium carbonate was obtained from Fisher Scientific, Loughborough, United Kingdom. NOA81 was obtained from Thorlabs, Dachau, Germany. All chemicals were obtained in highest available purity and used as received. The hydroxynitrile lyase from Manihot esculenta was a gift from Jülich Fine Chemicals (now Codexis). Alcohol dehydrogenase from Lactobacillus kefir (LkADH) and glucose dehydrogenase (GDH) were purchased from evocatal, Monheim, Germany. Deionized water was produced with an Ultra Clear Reinstwassersystem by SG Water (now Evoqua, Guenzburg, Germany) and used throughout this study.

\section{Analytical Methods}

Enzyme activity was measured with spectrophotometer Specord 50 from Analytik Jena, Jena, Germany. Conversion and enantiomeric excess' of all reactions were measured by gas chromatography with a Trace 1310 gas chromatograph (from Thermo Scientific, Dreieich, Germany) with a 1300 flame ionization detector equipped with a Chirasil-Dex-CB-column $(25 \mathrm{~m} \times 0.25 \mathrm{~mm} \times 0.25 \mu \mathrm{m})$. Carrier gas helium (purity: 

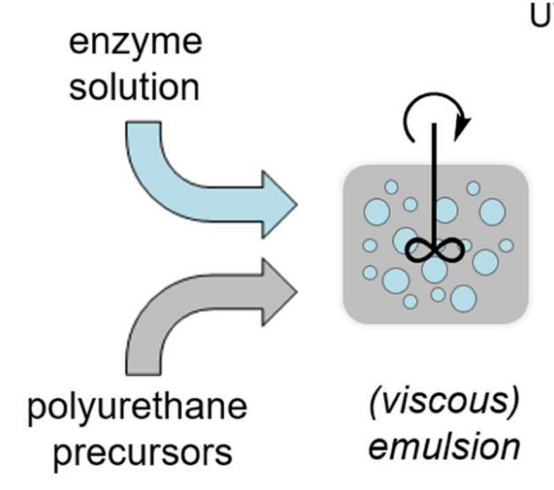

UV @ 320-400 nm

ca. $5 \mathrm{~min}$
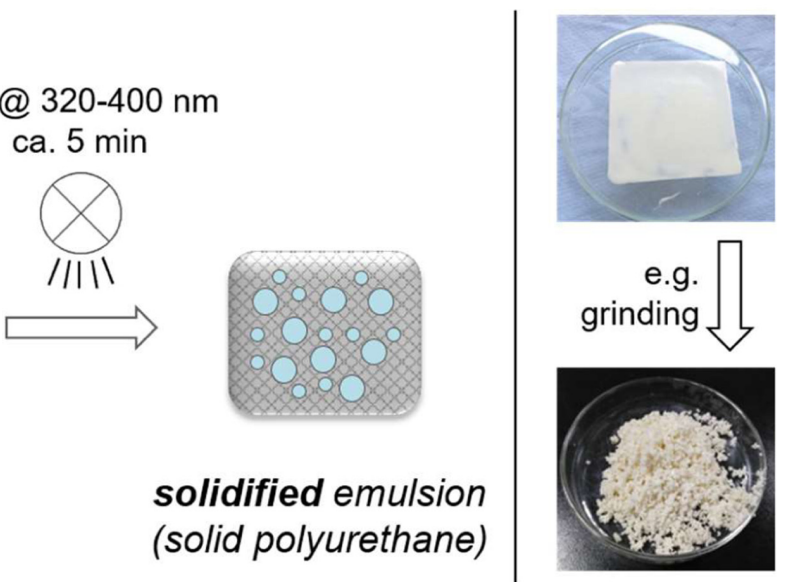

FIGURE 1 | General preparation of UV-cured enzyme compartments in polyurethane.

99.999\%) with a flow rate of $1.7 \mathrm{~mL} / \mathrm{min}$ was used throughout this study. Temperatures of the injector and detector were set to $250^{\circ} \mathrm{C}$. Temperature program: $60^{\circ} \mathrm{C}$ for $4 \mathrm{~min}$, followed by a heating rate of $5 \mathrm{~K} / \mathrm{min}$ to $140^{\circ} \mathrm{C}$. NMR spectra were recorded in $\mathrm{CDCl} 3$ with a Bruker Avance $250 \mathrm{MHz}$ (Rheinstetten, Germany).

\section{Enzyme Assays}

Alcohol dehydrogenase activity was determined by monitoring the consumption of NADPH at $340 \mathrm{~nm}$ over $3 \mathrm{~min}$ at $30^{\circ} \mathrm{C}$. Assay conditions: A standard reaction solution of $1 \mathrm{~mL}$ contained $970 \mu \mathrm{L}$ of $11 \mathrm{mM}$ acetophenone with $1 \mathrm{mM} \mathrm{MgCl} 2$ in $50 \mathrm{mM}$ TEA buffer $\mathrm{pH}$ 7, $20 \mu \mathrm{L} 12.5 \mathrm{mM} \mathrm{NADPH}$ and $10 \mu \mathrm{L}$ enzyme sample. Specific activity was $43.3 \cdot \mathrm{Umg}^{-1}$. Hydroxynitrile lyase activity was measured spectrophotometrically by monitoring the release of benzaldehyde from racemic benzaldehyde cyanohydrin (mandelonitrile) over $3 \mathrm{~min}$ at $280 \mathrm{~nm}$. Assay conditions: $25^{\circ} \mathrm{C}$, $900 \mu \mathrm{L} 50 \mathrm{mM}$ citrate buffer $\mathrm{pH} 5,50 \mathrm{~mL}$ stock solution $(100 \mathrm{mM})$ of rac-mandelonitrile (HNL) in $10 \mathrm{mM}$ citric acid solution and $50 \mu \mathrm{L}$ enzyme sample solution. Volumetric activity was $96 \mathrm{U} \cdot \mathrm{mL}^{-1}$. All measurements were executed in triplicate (error bars indicate standard deviation), and the spontaneous reaction subtracted. One unit of enzyme activity is defined as the conversion of $1 \mu \mathrm{mol}$ substrate per minute under assay conditions. Extinction coefficients: ADH-assay: $\varepsilon(340 \mathrm{~nm}$, $\mathrm{NADPH})=6220 \mathrm{~L} \cdot \mathrm{mol}^{-1} \cdot \mathrm{cm}^{-1}$ : HNL-assay: $\varepsilon(280 \mathrm{~nm}$, benzaldehyde) $=1352 \mathrm{~L} \cdot \mathrm{mol}^{-1} \cdot \mathrm{cm}^{-1}$.

\section{Compartmentalization of Enzymes}

For a typical immobilization $1 \mathrm{~g}$ NOA 81 were manually emulsified with $400 \mu \mathrm{L}$ of $5 \mathrm{mg} / \mathrm{mL}$ enzyme solution (containing also $1 \mathrm{mM}$ cofactor in $50 \mathrm{mM}$ phosphate or TEA buffer; in case of GDH: $100 \mathrm{mM}$ glucose and if required $20 \mathrm{mg} \mathrm{CaCO}_{3}$ ) for $1 \mathrm{~min}$, spread out to thickness of $1 \mathrm{~mm}$ and subsequently irradiated with UV-light $(366 \mathrm{~nm}$ ) for $5 \mathrm{~min}$ (no stirring). Irradiation was executed with a NU-4 UV Hand Lamp (4 W) by Herolab GmbH Laborgeräte (Wiesloch, Germany) at a distance of $12 \mathrm{~cm}$ in a closed container $(23 \mathrm{~cm} \times 18 \mathrm{~cm} \times 11 \mathrm{~cm})$. The obtained solid particles were cut into small pieces $(2-5 \mathrm{~mm}$ outer length) by a Cloer electric mill $(200 \mathrm{~W})$ (Cloer Elektrogeräte, Arnsberg, Germany) to facilitate a fast exchange of reactants.

\section{Microscopy}

The size distribution was directly measured from microscopic images of freshly cut polymeric materials. The images covered each an area of ca. $1.1 \mathrm{~mm}^{2}$. SEM analysis (scanning electron microscopy) was performed using the Supra-25 from Carl Zeiss AG, Jena, Germany. The Microscope was equipped with a field emission gun and InLens, SE, 4QBSD, EDX detectors. Before measurement, the samples were positioned on a wafer and coated with palladium/platinum layer (approximately $5 \mathrm{~nm}$ ). The analysis was run at $10 \mathrm{kV}$. Particle size was estimated using the Alicona MeX stereoscopic image analysis software.

\section{Hydrogen Cyanide Preparation}

All reactions including or forming hydrogen cyanide were performed in a well ventilated fume hood for self-protection. An electrochemical hydrogen cyanide detector Micro III G203, from GfG-Gesellschaft für Gerätebau, Dortmund, Germany was used throughout this study for continuous monitoring. The required amount of sodium cyanide was dissolved in ca. 10-fold amount of deionized water and cooled to $5^{\circ} \mathrm{C}$. Afterward $5 \mathrm{M}$ $\mathrm{HCl}$ was slowly added via a dropping funnel to the cyanide solution, while the internal temperature was kept below $10^{\circ} \mathrm{C}$ to avoid evaporation of hydrogen cyanide. After completion of the reaction the resulting solution was extracted twice with MTBE. The HCN-solution in MTBE was used for the compartmented biocatalytic cascade reaction without any further purification (see also below).

\section{General Reaction Procedure}

In a typical experiment the required amounts of acetophenone and 2-propanol were dissolved in $5 \mathrm{~mL}$ MTBE. Afterward the compartmented enzyme (see above) was added and the resulting suspension shaken horizontally at $30^{\circ} \mathrm{C}$ with an IKA HS $260 \mathrm{c}$ 
shaker at $180 \mathrm{rpm}$ (IKA-Werke, Staufen, Germany). In case of a two-enzyme cascade reaction the above mentioned HCNsolution (in MTBE) was used instead. The compartmented $\mathrm{MeHNL}$ was also added to the solution and the resulting mixture stirred at room temperature $\left(21^{\circ} \mathrm{C}\right)$ in a fume hood. Samples for reaction control and determination of enantiomeric excess (gas chromatography) were directly taken from the outer solvent. The measurements were executed in triplicate and the mean value presented (error bars indicate standard deviation).

\section{RESULTS AND DISCUSSION}

\section{Characterization of Aqueous Compartments}

The preparation of the polyurethane-based compartments is based on a simple two-step process. First, the hydrophobic polyurethane precursor material (NOA 81 by Norland Products) is vigorously mixed with an aqueous solution, which contains all required enzymes, cofactors, co-substrates, and buffer salts (Norland_Products, 2017). This results in a viscous water-inoil-emulsion with small independent droplets of the original aqueous phase. Due to the high viscosity of the precursor material an additional use of an emulsifier is not required. Subsequently curing of this mixture with UV-light eventually initiates polymerization of the polyurethane precursors, forming a solid, rubber-like material. The aqueous inclusions are not directly affected by polymerization and remain physically fixed as full medium inclusion in the polymer matrix. This phase includes all dissolved or otherwise suspended compounds in the aqueous phase, while polyurethane forms a physical barrier/membrane around it, which prevents any further coalescence of the aqueous droplets into a larger bulk phase. The dispersed aqueous inclusions appear randomly positioned within the solid polymer material. The distribution of the aqueous inclusions was visualized by dyeing the aqueous domains with bromophenol blue, while the polymer itself remains transparent (Figure 2A).
Air inclusions, originating from the preparation process, are occasionally found as 'empty' pockets in the polymer. REMimages of freshly cut preparations show clearly the highly spherical nature of these aqueous inclusions (Figure 2B). This behavior originates back to the uncured emulsion, which minimize interfacial tension by a low surface to volume ratio in such spherical form.

Up to $37.5 \mathrm{wt} \%$ of an aqueous phase can be incorporated into the final polymer preparation without any noticeable difference to polymer preparation without any included aqueous phases. Higher amounts of water causes insufficient curing to gel-like materials, which are not applicable as enzyme compartments. Also high protein concentrations do not affect curing of the emulsion. Up to $20 \mathrm{mg} / \mathrm{mL}$ of a model protein (bovine serum albumin) were incorporated without interfering with the curing process, which indicates the separation of the aqueous domains from the curing process within the polymer network. Noticeable leaching of protein from polyurethane compartments was not found, e.g., for $L k A D H$.

The actual size distribution of the aqueous inclusions is directly influenced by the stirring of the precursor/wateremulsion before UV-curing locks its size and position. Within this study the majority of inclusions are in a range of 5-75 $\mu \mathrm{m}$ with occasionally larger inclusions of $>100 \mu \mathrm{m}$ (Figure 3). Larger inclusions obviously hold more volume of an aqueous phase and thus more catalytic activity, but smaller domains provide a very high surface area for a fast exchange of reactants via its water-solid polymer-interface.

The obtained polymer preparations are meant to be used in organic solvents to ensure high solubilities for hydrophobic reactants. Therefore water-loaded polyurethane preparations were tested against various organic solvents with different polarities. All investigated organic solvents do not cause disintegration of the polymer matrix, but swelling occurs for a number of solvents. Strong swelling was found using DMSO and THF, moderate swelling with toluene and diethyl ether and no swelling was observed with MTBE, $n$-butanol, acetone, and

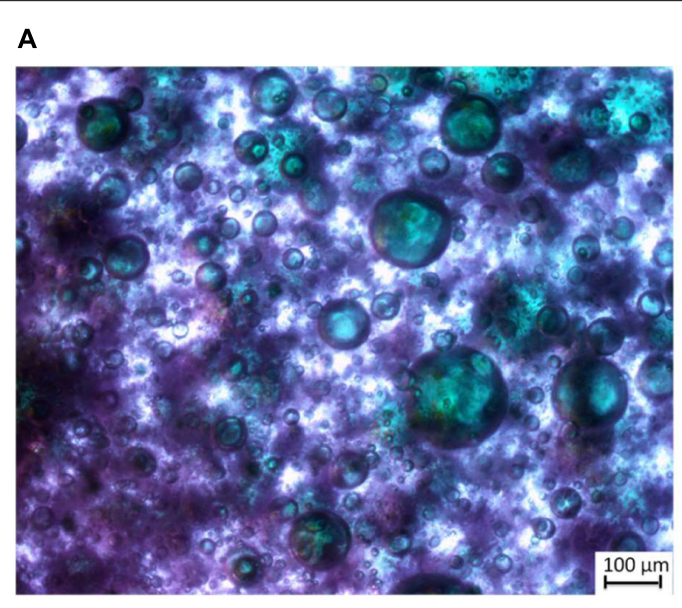

B

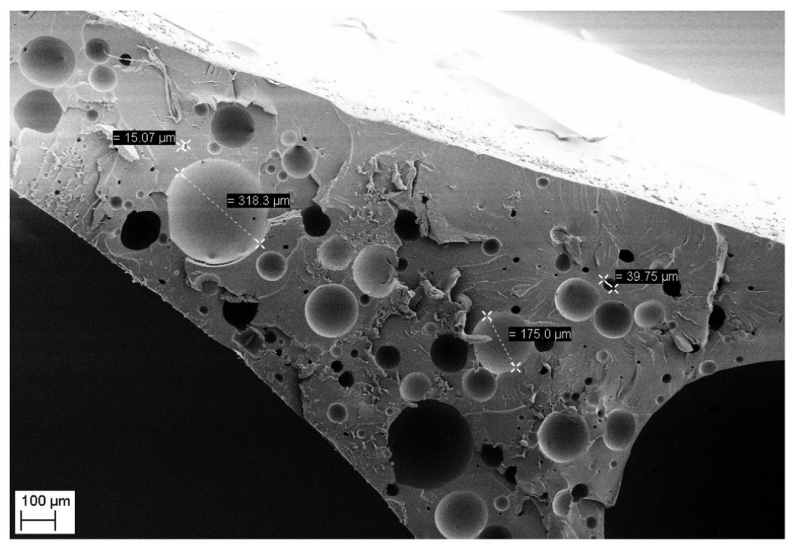

FIGURE 2 | Exemplary microscopic images of aqueous inclusions in a polyurethane matrix. (A) Light microscope image: spherical inclusions (highlighted blue/green by an aqueous solution of bromophenol blue) in the polyurethane matrix. (B) SEM image of a polyurethane cross section. 


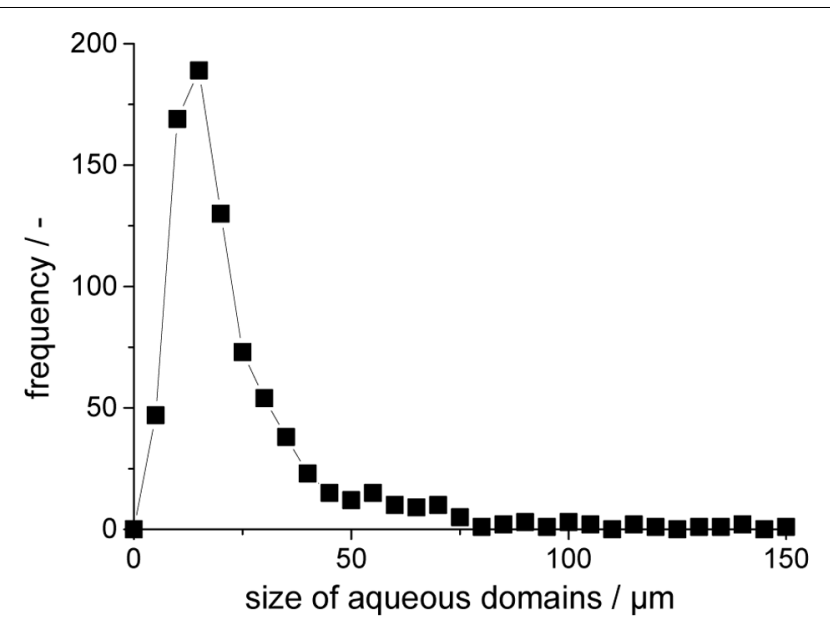

FIGURE 3 | Size distribution of aqueous inclusions in the polyurethane preparation.

ethanol. In addition, acidic solutions are easily tolerated by these compartment preparations, but strong bases will hydrolyze the polyurethane material very easily.

\section{Alcohol Dehydrogenase-Catalyzed Reaction}

The applicability of polyurethane-based compartments was investigated in detail for the reduction of prochiral ketones with the alcohol dehydrogenase from Lactobacillus kefir, LkADH. This includes cofactor regeneration by two commonly used regeneration concepts, using (a) co-substrate 2-propanol in a substrate-coupled approach (Figure 4A) and (b) D-glucose in an enzyme-coupled approach using glucose dehydrogenase (Figure 4B) (Eckstein et al., 2004; Kara et al., 2014).

In both cases cofactor regeneration takes place directly within the polyurethane compartments, consistent with the compartmentation concept. Simultaneously the final product (chiral alcohol) enriches throughout the reaction in the external

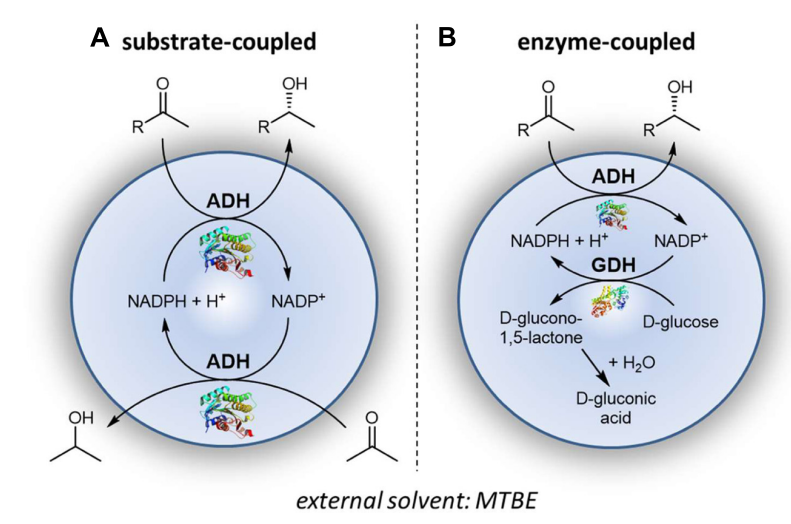

FIGURE 4 | Compartmentalized $\angle K A D H$-catalyzed reaction with (A) substrate coupled and (B) enzyme coupled cofactor regeneration. solvent phase. In case of the substrate-coupled approach coproduct acetone accumulates in the external solvent phase as well and can be removed from there without interfering with the enzyme, cofactor or water phase in general, e.g., by evaporation. A noteworthy exception is the use of glucose dehydrogenase for cofactor regeneration since D-glucose, D-glucono-1,5-lactone, and D-gluconic acid remain fully compartmentalized in the polyurethane preparation. The final co-product D-gluconic acid can be easily captured in the buffered solution or at co-compartmentalized calcium carbonate. In this case, only the desired chiral alcohol enriches in the external solvent phase.

Regardless of the applied cofactor regeneration concept similar reaction velocities were obtained with compartmented alcohol dehydrogenase from Lactobacillus kefir for the conversion of acetophenone (Figure 5A). The shown reaction velocity can be further improved by higher alcohol dehydrogenase concentrations (data not shown). This indicates again that $L k \mathrm{ADH}$ and the applied cofactor regeneration reaction are generally not affected by the polymerization procedure and fully compartmentalized. After curing the transport of reactants is solely achieved by diffusion through the cured polymer network into the aqueous inclusion. Swelling of polyurethane (see also above) by organic solvents also contributes to the transport phenomena, but seem to improve transport in general. Similarly other prochiral ketones can be easily converted by compartmentalized alcohol dehydrogenase with high enantioselectivities (Figure 5B). Herein especially smaller and more polar substrates are reduced very fast by compartmented $L k \mathrm{ADH}$, e.g., $80 \%$ equilibrium conversion for 2-pentanone was obtained within a few hours, while longer reaction times are required for 2-nonanone and even larger substrates. In comparison, a classical biphasic reaction system yields similar identical conversions (Eckstein et al., 2006), however, larger substrates are converted faster in comparison to compartmentalized $L k A D H$. This effect seems to be a combination of two main effects. First, diffusion limitation of large, unpolar reactants, while smaller, polar molecules such as 2-propanol and $(R)$-2-pentanol diffuse rather fast through the polyurethane membrane. Second, unpolar substrates such as 2-dodecanone partition into the external organic solvent and also unpolar polymer material, which results in a lower substrate concentration in the compartmented aqueous domain, e.g., even below the respective $K_{\mathrm{M}}$-value, which yields lower apparent activities for such large unpolar substrates.

In addition, the stability of the obtained enzyme compartments was investigated to ensure sufficient applicability also after prolonged storage times. Reaction time courses after given time periods were obtained since samples for classical spectrophotometric measurements cannot be taken from the solidified compartmentalized aqueous inclusions.

As shown in Figure 6A, at $4^{\circ} \mathrm{C}$ only a small loss of enzyme activity was observed ( $<20 \%$ after 14 days), which is shown as a slightly lower initial reaction rate. However, sufficient enzyme activity is preserved and equilibrium conversion was also reached with 14 days old samples. Lower temperature improve enzyme stability even further and at $-18^{\circ} \mathrm{C}$ only 

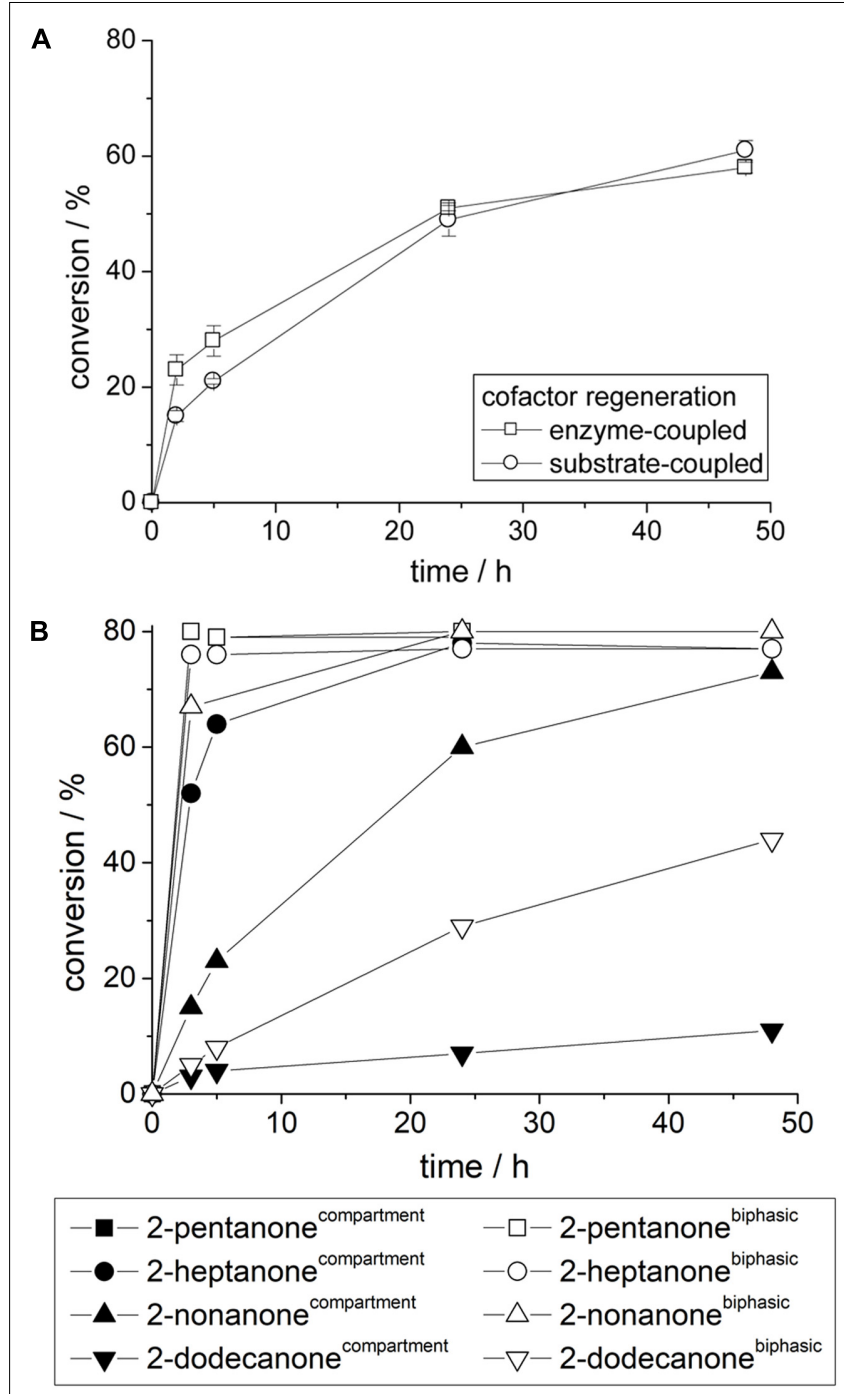

FIGURE 5 | (A) Reaction of compartmented LKADH with enzyme-coupled and substrate-coupled cofactor regeneration. (B) Comparison of compartmented $\angle K A D H$ and a classical biphasic system for the conversion of various prochiral aliphatic ketones; Reaction conditions: Compartmented LkADH: 10 mM substrate, 100 mM 2-propanol in 5 mL MTBE (external solvent); biphasic reaction system: $10 \mathrm{mM}$ substrate, $100 \mathrm{mM}$ 2-propanol in $1 \mathrm{~mL}$ MTBE and $4 \mathrm{~mL} 50 \mathrm{mM}$ phosphate buffer $\mathrm{pH} 7.5$ (incl. identical amount of dissolved $L k A D H)$.

a marginal loss of enzyme activity was seen after 7 and 14 days of storage (Figure 6B). These results are consistent with stability measurements of a (classical) aqueous solution of $L k \mathrm{ADH}$ at similar conditions, which highlights again the full compartmentalization of the entire aqueous phase. Unwanted secondary effects such as byproducts from curing or the negative side effect from the present hydrophobic surface were not observed.

\section{Model Cascade Reaction}

As a model reaction a combination of two very contrary reaction systems was chosen, the alcohol dehydrogenase from
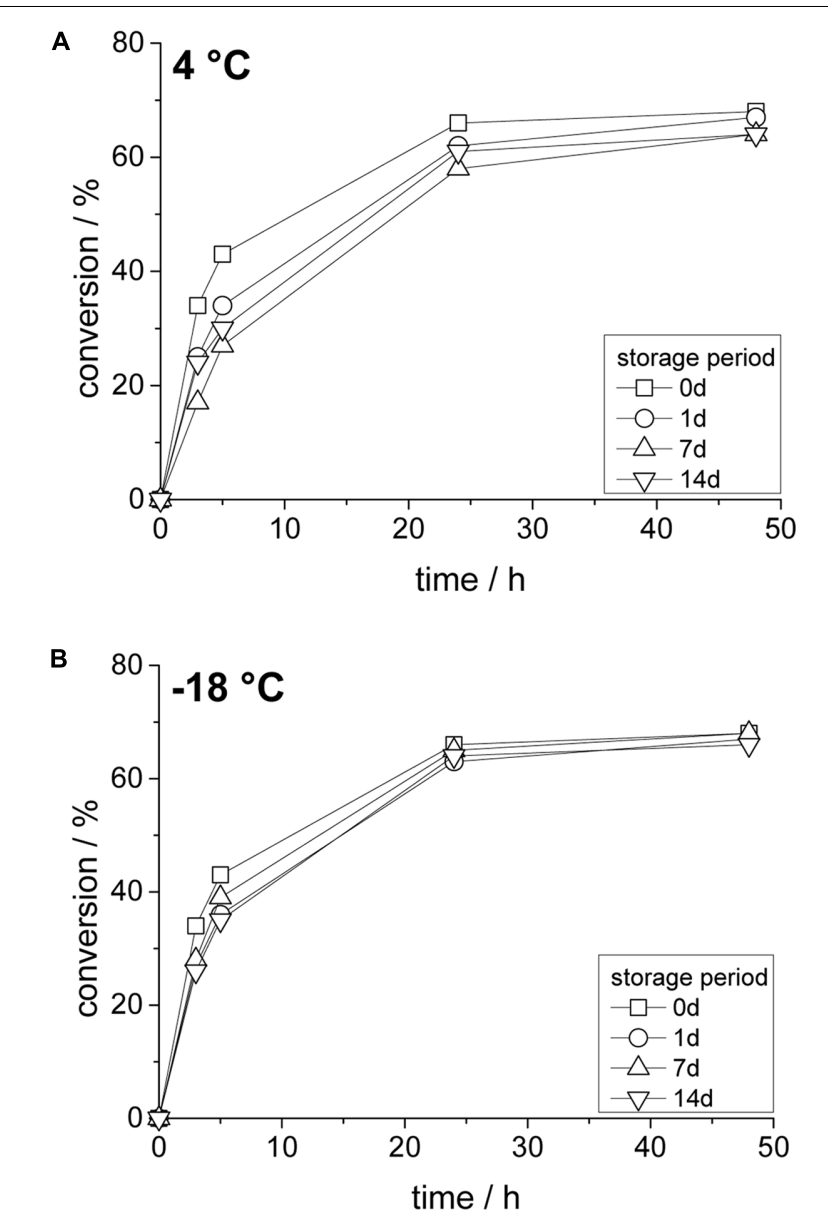

FIGURE 6 | Effect of storage temperature and time on compartmented $\angle K A D H$ with substrate-coupled cofactor regeneration; (A) storage at $4^{\circ} \mathrm{C}$, (B) storage at $-18^{\circ} \mathrm{C}$. Reaction conditions: $10 \mathrm{mM}$ substrate in $5 \mathrm{~mL}$ MTBE, $100 \mathrm{mM}$ 2-propanol (in external solvent).

Lactobacillus kefir, LkADH (see also above) and hydroxynitrile lyase from Manihot esculenta, MeHNL (Figure 7). The shown example is intended as a model concept for biocatalytic systems, including alcohol dehydrogenases, with incompatible reaction requirements.

In reaction compartment A prochiral acetophenone is converted by $L k A D H$ to $(R)-1$-phenylethanol, which enriches in the external solvent phase. The oxidized cofactor $\mathrm{NADP}^{+}$is simultaneously regenerated to $\mathrm{NADPH}+\mathrm{H}^{+}$by a substratecoupled approach with 2-propanol yielding acetone (see also above). Acetone is then subsequently converted at a different $\mathrm{pH}$ to acetone cyanohydrin in reaction compartment B by $\mathrm{MeHNL}$, representing principally an in situ-product removal (ISPR) of the co-product acetone.

The reaction requirements of these two biocatalysts are unfortunately significantly different. Alcohol dehydrogenasecatalyzed reductions of carbonyl compounds are typically performed at neutral $\mathrm{pH}$-conditions and moderate reaction temperatures, e.g., $30^{\circ} \mathrm{C}$. $\mathrm{MeHNL}$ on the other hand has its optimal reaction parameters at $\mathrm{pH} 5.5$ and $60^{\circ} \mathrm{C}$ (Andexer 


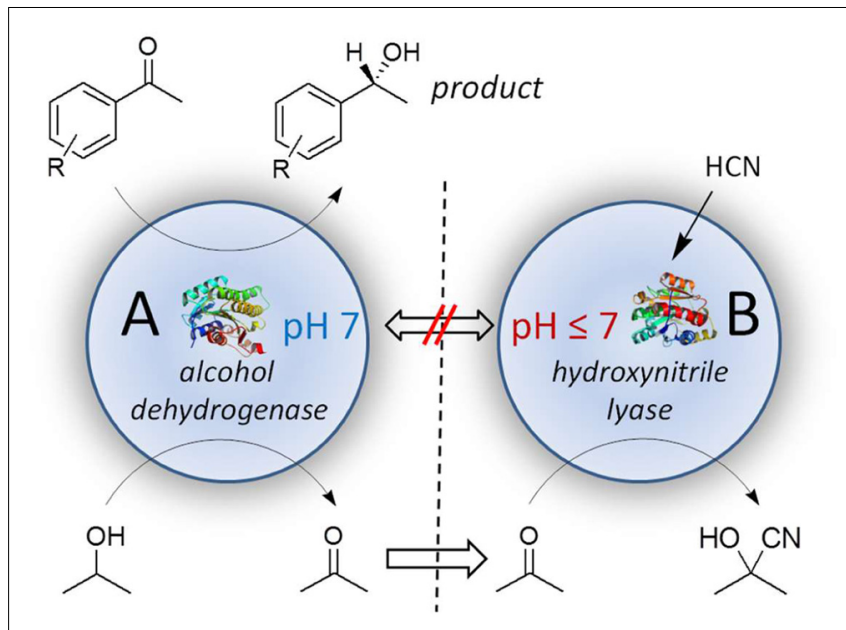

FIGURE 7 | Compartmented cascade reaction with $\angle K A D H$ in compartment $A$ at $\mathrm{pH} 7$ and $\mathrm{MeHNL}$ in compartment $\mathrm{B}$ at $\mathrm{pH}<7$.

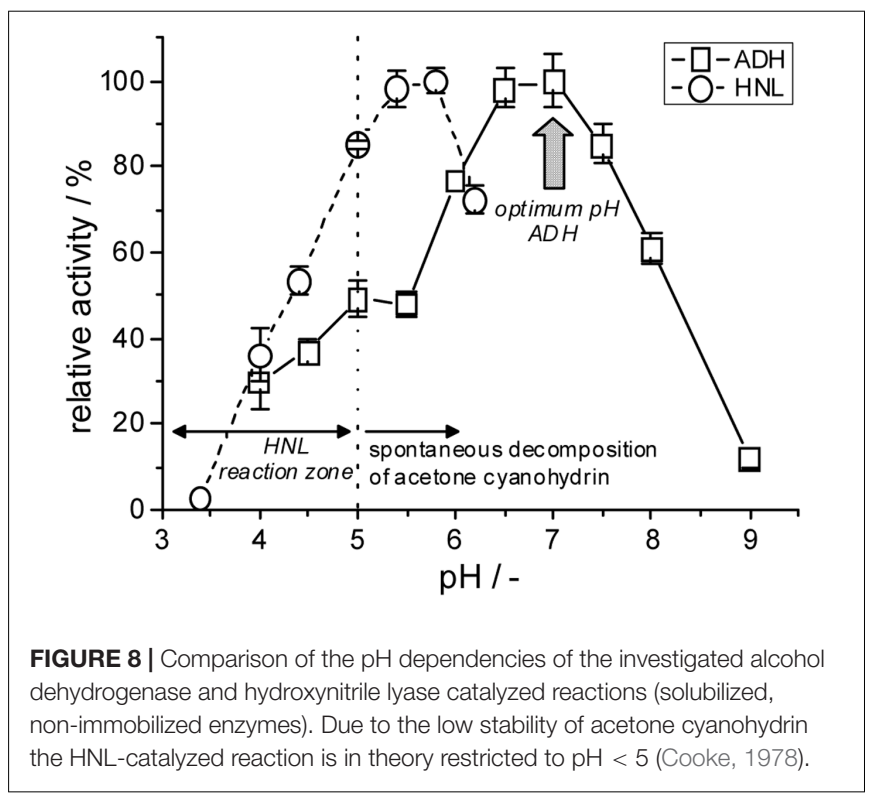

et al., 2009; Guterl et al., 2009) (Figure 8). However, HNLs are generally used significantly below these parameters, e.g., in a $\mathrm{pH}$ range of 3-5 and temperatures below $10^{\circ} \mathrm{C}$, which is required to suppress product racemization and decomposition of the respective cyanohydrin products (Von Langermann et al., 2008; Avi et al., 2009).

The shown compartmentation concept overcomes these problems by using two different polymer matrices each with specifically chosen optimal reaction parameters. $L k \mathrm{ADH}$ was compartmented at $\mathrm{pH} 7$, while the hydroxynitrile lyase remained at acidic conditions between $\mathrm{pH} 3$ and 7 . The relevant reactants, however, diffuse via the external solvent between the compartments (Figure 7) and are converted by the included biocatalysts. This reaction concept can be described as a multiphase-reaction system (external solvent, solid polymer

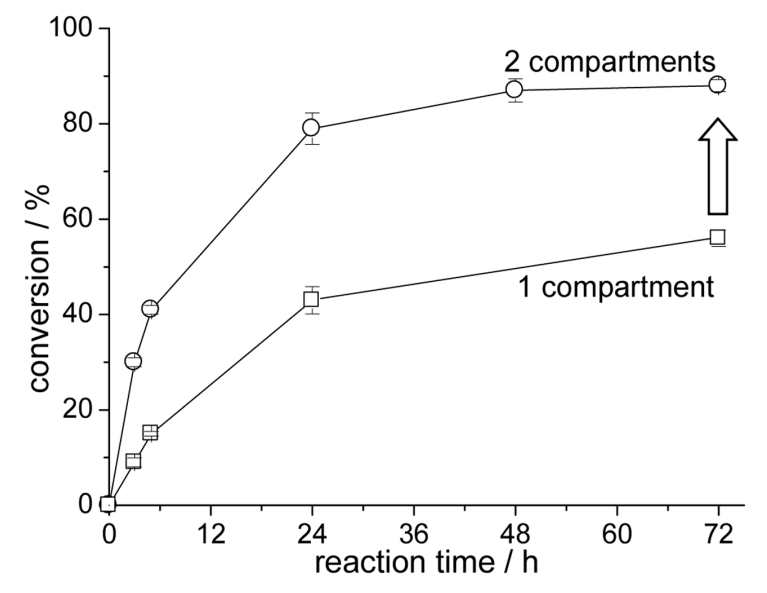

FIGURE 9 | LKADH-MeHNL cascade reaction with one or two compartmented reaction zones; 1 compartment: only $L k A D H$-compartments (open squares), 2 compartments: $\angle K A D H$-compartments at $\mathrm{pH} 7$ and MeHNL-compartments at $\mathrm{pH} 5$ (open circles); reaction conditions: $30^{\circ} \mathrm{C}$, $5 \mathrm{~mL}$ MTBE, $10 \mathrm{mM}$ acetophenone, $100 \mathrm{mM}$ 2-propanol and $200 \mathrm{mM}$ hydrogen cyanide for the 2 compartment-reaction, enantiomeric excess of (R)-1-phenylethanol $\geq 99 \%$.

phases, included aqueous domains in the compartments), which are interconnected by multiple diffusion systems.

Moreover, the chosen low water-content of polyurethanebased compartmentation is also relevant for the intended synthesis of acetone cyanohydrin (shown in Figure 7), which easily decompose in aqueous solutions, especially at $\mathrm{pH}>5$. Thus a hypothetical combination of both enzymes in an aqueous system is limited to $\mathrm{pH}<5$ and temperature of $<10^{\circ} \mathrm{C}$, which is not feasible for the applied alcohol dehydrogenase $L k A D H$. Fortunately this is avoided by compartmentation, which significantly reduces these undesired side reactions by a full removal of overall water, except its minimum usage within the compartments for the enzyme (Von Langermann and Wapenhensch, 2014). Herein HNLs can now be used at optimal $\mathrm{pH}$, higher temperatures and without loss of the desired cyanohydrin.

As a result higher equilibrium conversions are obtained for the initial reduction reaction of acetophenone (Figure 9). The single compartmentation of the alcohol dehydrogenasecatalyzed $(\mathrm{pH} 7)$ reaction yields a conversion of only $56 \%$ after $72 \mathrm{~h}$ (filled triangle). The addition of the second hydroxynitrile lyase reaction compartment for the simultaneous synthesis of acetone cyanohydrin increased the conversion of the initial reduction reaction to $88 \%$. This represents a significant improvement in comparison to the single compartmented enzyme reaction. In a side reaction acetophenone is also converted to $(S)$-acetophenone cyanohydrin by $M e H N L$ in reaction compartment $\mathrm{B}$, which fortunately only reduces the overall yield of the alcohol dehydrogenase-catalyzed reaction by a very small amount (von Langermann et al., 2007). In addition, similarly to a single compartmented $L k \mathrm{ADH}$-catalyzed reaction the ratio of prochiral ketone and 2-propanol, as part of the alcohol dehydrogenase-catalyzed reaction, affects the 
equilibrium conversion within the cascade reaction (Eckstein et al., 2006).

\section{SUMMARY AND CONCLUSION}

In the presented work, the preparation and characterization of polyurethane-based materials for the compartmentation of enzymes and their subsequent use for biocatalytic reactions was investigated. The shown enzyme compartments were easily obtained by UV-light curing of an emulsion of a hydrophobic polyurethane precursor and an aqueous enzyme solution. Based on the formed initial emulsion the incorporated aqueous domains of the enzyme solution remain stable and finely dispersed in the polymer matrix, which itself is stable against various solvents.

As shown in this study, such a polymer-based compartmentation technique is compatible with alcohol dehydrogenase-, glucose dehydrogenase-, and hydroxynitrile lyase-catalyzed reaction systems. Negative effects for the included enzymes from the curing process or presence of the hydrophobic polymer phase (e.g., phase boundary within the polymer preparation) were not observed. The general synthetic applicability was shown for a single alcohol dehydrogenasecatalyzed reaction, enabling high conversions and enantiomeric excess of the respective chiral alcohols. In addition, different enzyme compartments with contrary reaction requirements can be used simultaneously in one reaction vessel, which facilitates

\section{REFERENCES}

Abu, R., and Woodley, J. M. (2015). Application of enzyme coupling reactions to shift thermodynamically limited biocatalytic reactions. ChemCatChem 7 , 3094-3105. doi: 10.1002/cctc.201500603

Andexer, J. N., Langermann, J. V., Kragl, U., and Pohl, M. (2009). How to overcome limitations in biotechnological processes - examples from hydroxynitrile lyase applications. Trends Biotechnol. 27, 599-607. doi: 10.1016/j.tibtech.2009.07.005

Ansorge-Schumacher, M. B. (2007). Two-phase systems with solidified water phases - tools for technical use of sensitive catalysts. Mini Rev. Org. Chem. 4, 243-245. doi: 10.2174/157019307781369940

Avi, M., Griengl, H., Roberge, C., Fleitz, F., Devine, P., Langermann, J. V., et al. (2009). "Synthesis of cyanohydrins using hydroxynitrile lyases", in Practical Methods for Biocatalysis and Biotransformations (Hoboken, NJ: John Wiley \& Sons), 255-258. doi: 10.1002/9780470748589.ch8

Barabási, A. L., and Oltvai, Z. N. (2004). Network biology: understanding the cell's functional organization. Nat. Rev. Genet. 5, 101-113. doi: 10.1038/nrg1272

Buthe, A., Kapitain, A., Hartmeier, W., and Ansorge-Schumacher, M. B. (2005). Generation of lipase-containing static emulsions in silicone spheres for synthesis in organic media. J. Mol. Catal. B Enzym. 35, 93-99. doi: 10.1016/j. molcatb.2005.05.006

Cabirol, F. L., Hanefeld, U., and Sheldon, R. A. (2006). Immobilized hydroxynitrile lyases for enantioselective synthesis of cyanohydrins: sol-gels and cross-linked enzyme aggregates. Adv. Synth. Catal. 348, 1645-1654. doi: 10.1002/adsc. 200606139

Carlborg, C. F., Haraldsson, T., Öberg, K., Malkoch, M., and Van Der Wijngaart, W. (2011). Beyond PDMS: off-stoichiometry thiol-ene (OSTE) based soft lithography for rapid prototyping of microfluidic devices. Lab Chip 11, 3136-3147. doi: 10.1039/c1lc20388f

Cooke, R. D. (1978). An enzymatic assay for the total cyanide content of cassava (Manihot esculenta Crantz). J. Sci. Food Agric. 29, 345-352. doi: 10.1002/jsfa. 2740290408 the use of enzymatic cascade reaction in separated reaction zones with different reaction conditions. This includes also practically incompatible reaction conditions, as shown in the combined alcohol dehydrogenase-hydroxynitrile lyase-cascade reaction. Moreover, the shown polyurethane-based concept can be easily transferred to other (bio)catalytic reaction systems and further cascade reactions, especially with similar incompatible reaction requirements.

\section{AUTHOR CONTRIBUTIONS}

DU and JvL conceived and designed the study. DU performed the experiments. DU and JvL analyzed the data and prepared the manuscript.

\section{FUNDING}

Financial support by the German Federal Ministry of Education and Research (BMBF, Bundesministerium für Bildung and Forschung, Project No. 031A123) is highly acknowledged.

\section{ACKNOWLEDGMENT}

The authors thank Jan C. Peinemann (University of Rostock) for his assistance in the experimental work.

Eckstein, M., Daußmann, T., and Kragl, U. (2004). Recent developments in $\mathrm{NAD}(\mathrm{P}) \mathrm{H}$ regeneration for enzymatic reductions in one- and twophase system. Biocatal. Biotransformation 22, 89-96. doi: 10.1080/ 10242420410001692769

Eckstein, M. F., Peters, M., Lembrecht, J., Spiess, A. C., and Greiner, L. (2006). Maximise equilibrium conversion in biphasic catalysed reactions: mathematical description and practical guideline. Adv. Synth. Catal. 348, 1591-1596. doi: 10.1002 /adsc.200606079

Faber, K. (2004). Biotransformations in Organic Chemistry. Berlin: Springer. doi: 10.1007/978-3-642-18537-3

Gelman, F., Blum, J., and Avnir, D. (2002). One-pot sequences of reactions with sol-gel entrapped opposing reagents: an enzyme and metal-complex catalysts. J. Am. Chem. Soc. 124, 14460-14463. doi: 10.1021/ja020799+

Guterl, J. K., Andexer, J. N., Sehl, T., von Langermann, J., Frindi-Wosch, I., Rosenkranz, T., et al. (2009). Uneven twins: comparison of two enantiocomplementary hydroxynitrile lyases with $\alpha / \beta$-hydrolase fold. J. Biotechnol. 141, 166-173. doi: 10.1016/j.jbiotec.2009.03.010

Guterl, J. K., and Sieber, V. (2013). Biosynthesis "debugged": novel bioproduction strategies. Eng. Life Sci. 13, 4-18. doi: 10.1002/elsc.201100231

Huh, W. K., Falvo, J. V., Gerke, L. C., Carroll, A. S., Howson, R. W., Weissman, J. S., et al. (2003). Global analysis of protein localization in budding yeast. Nature 425, 686-691. doi: 10.1038/nature02026

Kara, S., Schrittwieser, J. H., Hollmann, F., and Ansorge-Schumacher, M. B. (2014). Recent trends and novel concepts in cofactor-dependent biotransformations. Appl. Microbiol. Biotechnol. 98, 1517-1529. doi: 10.1007/s00253-0135441-5

Klermund, L., Poschenrieder, S. T., and Castiglione, K. (2017). Biocatalysis in polymersomes: improving multienzyme cascades with incompatible reaction steps by compartmentalization. ACS Catal. 7, 3900-3904. doi: 10.1021/acscatal. $7 \mathrm{~b} 00776$

Köhler, V., and Turner, N. J. (2014). Artificial concurrent catalytic processes involving enzymes. Chem. Commun. 51, 450-464. doi: 10.1039/c4cc07277d 
Muschiol, J., Peters, C., Oberleitner, N., Mihovilovic, M., Bornscheuer, U., and Rudroff, F. (2015). Cascade catalysis - strategies and challenges en route to preparative synthetic biology. Chem. Commun. 51, 5798-5811. doi: 10.1039/ c4cc08752f

Norland_Products (2017). NOA 81 Safety Data Sheet. Available: https://www. norlandprod.com $/ \mathrm{msds} /$ noa\%2081msd.html

Oberleitner, N., Peters, C., Muschiol, J., Kadow, M., Saß, S., Bayer, T., et al. (2013). An enzymatic toolbox for cascade reactions: a showcase for an in vivo redox sequence in asymmetric synthesis. ChemCatChem 5, 3524-3528. doi: 10.1002/ cctc. 201300604

Oroz-Guinea, I., and García-Junceda, E. (2013). Enzyme catalysed tandem reactions. Curr. Opin. Chem. Biol. 17, 236-249. doi: 10.1016/j.cbpa.2013.02.015

Ovádi, J., and Saks, V. (2004). On the origin of intracellular compartmentation and organized metabolic systems. Mol. Cell. Biochem. 256-257, 5-12. doi: 10.1023/B: MCBI.0000009855.14648.2c

Patterson, D. P., Schwarz, B., Waters, R. S., Gedeon, T., and Douglas, T. (2014). Encapsulation of an enzyme cascade within the bacteriophage P22 virus-like particle. ACS Chem. Biol. 9, 359-365. doi: 10.1021/cb40 06529

Pierre, A. C. (2004). The sol-gel encapsulation of enzymes. Biocatal. Biotransformation 22, 145-170. doi: 10.1080/10242420412331283314

Ricca, E., Brucher, B., and Schrittwieser, J. H. (2011). Multi-enzymatic cascade reactions: Overview and perspectives. Adv. Synth. Catal. 353, 2239-2262. doi: 10.1002/adsc.201100256

Riva, S., and Fessner, W.-D. (2014). Cascade Biocatalysis Integrating Stereoselective and Environmentally Friendly Reactions. Weinheim: Wiley-VCH Verlag $\mathrm{GmbH} \& \mathrm{Co}$.

Sánchez-Moreno, I., Oroz-Guinea, I., Iturrate, L., García-Junceda, E., Carreira, E. M., and Yamamoto, H. (2012). "7.20 multi-enzyme reactions," in Comprehensive Chirality, eds H. Yamamoto and E. M. Carreira (Amsterdam: Elsevier), 430-453.

Schrittwieser, J. H., Sattler, J., Resch, V., Mutti, F. G., and Kroutil, W. (2011). Recent biocatalytic oxidation-reduction cascades. Curr. Opin. Chem. Biol. 15, 249-256. doi: 10.1016/j.cbpa.2010.11.010

Simon, R. C., Richter, N., Busto, E., and Kroutil, W. (2014). Recent developments of cascade reactions involving $\omega$-transaminases. ACS Catal. 4, 129-143. doi: $10.1021 / \operatorname{cs} 400930 \mathrm{v}$
Tao, J., and Kazlauskas, R. (2011). Biocatalysis for Green Chemistry and Chemical Process Development. Hoboken, NJ: John Wiley \& Sons. doi: 10.1002/ 9781118028308

Tu, B. P., Kudlicki, A., Rowicka, M., and McKnight, S. L. (2005). Cell biology: Logic of the yeast metabolic cycle: temporal compartmentalization of cellular processes. Science 310, 1152-1158. doi: 10.1126/science.1120499

Von Langermann, J., Guterl, J. K., Pohl, M., Wajant, H., and Kragl, U. (2008). Hydroxynitrile lyase catalyzed cyanohydrin synthesis at high $\mathrm{pH}$-values. Bioprocess Biosyst. Eng. 31, 155-161. doi: 10.1007/s00449-008-0198-4

von Langermann, J., Mell, A., Paetzold, E., Daussmann, T., and Kragl, U. (2007). Hydroxynitrile lyase in organic solvent-free systems to overcome thermodynamic limitations. Adv. Synth. Catal. 349, 1418-1424. doi: 10.1002/ adsc. 200700016

Von Langermann, J., and Wapenhensch, S. (2014). Hydroxynitrile lyase-catalyzed synthesis of enantiopure cyanohydrins in biocatalytic active static emulsions (BASE) with suppression of the non-enzymatic side reaction. Adv. Synth. Catal. 356, 2989-2997. doi: 10.1002/adsc.201301149

Wu, C., Kraume, M., and Ansorge-Schumacher, M. B. (2011). Optimized biocatalytically active static emulsions for organic synthesis in nonaqueous media. ChemCatChem 3, 1314-1319. doi: 10.1002/cctc.201100085

Zecchin, A., Stapor, P. C., Goveia, J., and Carmeliet, P. (2015). Metabolic pathway compartmentalization: an underappreciated opportunity? Curr. Opin. Biotechnol. 34, 73-81. doi: 10.1016/j.copbio.2014.11.022

Zhang, L., Shi, J., Jiang, Z., Jiang, Y., Qiao, S., Li, J., et al. (2011). Bioinspired preparation of polydopamine microcapsule for multienzyme system construction. Green Chem. 13, 300-306. doi: 10.1039/c0gc00432d

Conflict of Interest Statement: The authors declare that the research was conducted in the absence of any commercial or financial relationships that could be construed as a potential conflict of interest.

Copyright (c) 2017 Uhrich and von Langermann. This is an open-access article distributed under the terms of the Creative Commons Attribution License (CC BY). The use, distribution or reproduction in other forums is permitted, provided the original author(s) or licensor are credited and that the original publication in this journal is cited, in accordance with accepted academic practice. No use, distribution or reproduction is permitted which does not comply with these terms. 